\title{
Magnetic resonance diffusion tensor imaging for occult lesion detection in multiple sclerosis
}

\author{
JIAFENG CHEN $^{1 *}$, CHUNKUI ZHOU $^{1 *}$, LIJUN ZHU $^{2}$, XIULI YAN ${ }^{1}$, \\ YONGHONG WANG $^{1}, \mathrm{XIN} \mathrm{CHEN}^{1}$ and SHAOKUAN FANG ${ }^{1}$ \\ ${ }^{1}$ Department of Neurology, Neuroscience Centre, The First Teaching Hospital of Jilin University, Changchun, Jilin 130021; \\ ${ }^{2}$ Department of Neurology, The Third Teaching Hospital of Jilin University, Changchun, Jilin 130041, P.R. China
}

Received September 12, 2015; Accepted October 5, 2016

DOI: $10.3892 / \mathrm{etm} .2016 .3950$

\begin{abstract}
It remains challenging to locate occult lesions in patients with multiple sclerosis (MS). Diffusion tensor imaging (DTI) has been demonstrated to have the potential to identify occult changes in MS lesions. The present study used 3.0T magnetic resonance DTI to investigate the characteristics of different stages of MS lesions. DTI parameters, fractional anisotropy (FA), mean diffusivity (MD), $\lambda / /$ and $\lambda \perp$ values of lesions were compared at the different stages of 10 patients with MS with 10 normal controls. The results demonstrated that FA and $\lambda / /$ values of MS silent and subacute lesions are decreased and MD and $\lambda \perp$ values are increased, as compared with those of normal appearing white matter (NAWM) and normal controls. NAWM FA values were lower, and MD, $\lambda / /$, and $\lambda \perp$ values were higher than those of normal controls. It was also indicated that MS lesions had reduced color signals compared with the controls, and the lesion area appeared larger using DTI as compared with diffusion-weighted imaging. Furthermore, fiber abnormalities were detected in MS lesions using DTT, with fewer fibers connected to the lesion side, as compared with the contralateral side. FA, MD, $\lambda / /$ and $\lambda \perp$ values in the thalamus were increased, as compared with those of normal controls $(\mathrm{P}<0.05)$; whereas $\mathrm{MD}, \lambda / /$ and $\lambda \perp$ values were significantly increased and FA values significantly decreased in the caudate nucleus and deep brain gray matter (DBGM) of patients with MS, as compared with the controls $(\mathrm{P}<0.05) . \lambda / /$ and $\lambda \perp$ values were also significantly increased in the DBGM of patients with MS as compared with normal controls $(\mathrm{P}<0.05)$. The present findings demonstrate that DTI may be useful in the characterization of MS lesions.
\end{abstract}

Correspondence to: Professor Shaokuan Fang, Department of Neurology, Neuroscience Centre, The First Teaching Hospital of Jilin University, 71 Xinmin Street, Changchun, Jilin 130021, P.R. China E-mail: fang20063536@sina.com

*Contributed equally

Key words: multiple sclerosis, diffusion tensor imaging, diffusion tensor tractography, deep gray matter, thalamus

\section{Introduction}

Multiple sclerosis (MS) is the most common inflammatory demyelinating disorder of the central nervous system (CNS) with a predilection for the optic nerves, corpus callosum, brainstem, spinal cord, cerebellum, and periventricular white matter $(1,2)$. It is the second most common neurological disorder within the northern hemisphere in young adults, and it is characterized by either a relapsing-remitting or progressive course (3). MS lesions can be identified by conventional magnetic resonance imaging (MRI); however, it remains challenging to detect lesions in early stage or occult MS lesions. Because of this, clinicians are unable to connect all clinical symptoms with the findings on MRI (4). Advanced MRI techniques, in particular, diffusion tensor imaging (DTI), have been demonstrated to have the potential to identify changes in MS lesions at earlier stages, including in normal appearing white matter. DTI can also be used for describing biological tissue microstructures, which exploit the quantification of water diffusion in tissues containing MS lesions (5). Moreover, DTI is able to measure the degree of diseased white matter more accurately than T2-weighted imaging, and may also detect abnormalities earlier than T2-weighted fast spin-echo imaging (5). DTI parameters, including fractional anisotropy (FA), mean diffusivity (MD), $\lambda \perp$ and $\lambda / /$, may represent important indicators of neuronal structure and its loss in patients suffering from MS.

To date, few studies have used DTI to compare different MS stages or used this technique to compare diseased tissue with normal appearing white matter (NAWM); the majority of studies have used a $1.5 \mathrm{~T}$ MRI for research. The present study used 3.0 T MR DTI technology to further study the pathophysiology of the different stages of MS lesions, NAWM and normal appearing deep gray matter (NADGM), and compared these values to normal controls. The findings of this study may help advance the early diagnosis, treatment and prognosis of MS in the future.

\section{Materials and methods}

Subjects. The present study was approved by the Ethics Committee of The First Teaching Hospital of Jilin University (Changchun, China), and written informed consent was obtained from all patients. 
Ten patients (female, 8 ; male, 2) and ten sex- and age-matched, right-handed, healthy controls were enrolled in the present study between September 2011 to June 2012. Patients were aged 27-55 years (mean, 37.3 years). All patients were diagnosed with definite MS according to McDonald criteria (6), and all patients had relapsing-remitting MS. Eight patients exhibited lesions in both the brain and spinal cord, whereas the remaining two only had brain lesions.

MRI scans. MRI studies were performed on a Trio Tim 3 T MRI scanner (Siemens AG, Munich, Germany), using a twelve-channel coil as a phased-array receiver and a body coil for transmission. Prior to the DTI scan, all patients were told to minimize eye movements.

T1-weighted MRIs were obtained with the following parameters: Repetition time (TR), $440 \mathrm{msec}$; Echo time (TE), $2.46 \mathrm{msec}$; TI, $900 \mathrm{msec}$; slice thickness, $5 \mathrm{~mm}$; matrix size, 256x320; field of view (FOV), 220x220 m²; and angle, $130^{\circ}$. T2-weighted MRIs were acquired with the following parameters: TR, 5,000 msec; TE, $93 \mathrm{msec}$; slice thickness, $5 \mathrm{~mm}$; matrix size, 320x320; interlayer spacing, $1.5 \mathrm{~mm}$; and FOV, 220x220 $\mathrm{mm}^{2}$. DTI scans were performed using the following parameters: TR, 5,000 msec; TE, $97 \mathrm{msec}$; b, 0 and $1,000 \mathrm{sec} / \mathrm{mm}^{3}$; slice thickness, $3 \mathrm{~mm}$; matrix size, 128x128; scan time, 5 min $42 \mathrm{sec}$; and diffusion gradient directions number, 64.

DTI measurements. DTIs can be characterized by a tensor ellipsoid, from which MD and FA values can be calculated. MD is a measure of the magnitude of molecular diffusion, whereas FA represents the degree of directionality of diffusion, which indicates the preference for a single direction of diffusion. When $F A=0$, this indicates isotropic diffusion, which is equal in all directions, and when $F A=1$, this indicates that diffusion occurs only along one axis. Notably, when axons or myelin are disrupted, FA values decrease, indicating that diffusion is not restricted only one direction. Both MD and FA values can reveal subtle, but definite, pathological changes that cannot be visualized with conventional T2-weighted or T1-weighted MRI, particularly in NAWM (7).

Another important measure analyzed in the present study was the average diffusion coefficient (ADC), which estimates total diffusion for each voxel analyzed. The magnitude of axial $(\lambda 1)$ and radial diffusivity $(\lambda 3, \lambda 2)$ was also determined. ADC increases if biological tissues are affected, indicating an increase in radial diffusion due to fewer diffusion hindrances. In white matter, water diffuses preferentially along axons and nerve fiber bundles (axial diffusivity, $\lambda 1$ ). The diffusion process is hindered in the perpendicular direction from the fibers by axonal membranes and is modulated by myelin. This radial diffusivity is reflected by $\lambda 2 \& \lambda 3$. Usually, $\lambda \perp=(\lambda 2+\lambda 3) / 2$, which reflects water diffusion process in the perpendicular direction, while $\lambda /=\lambda 1$, and reflects water diffusion in the parallel direction (5). The directionally-encoded color (DEC) map directly visualizes the fiber and lesions.

DTI analysis. DTI acquisitions were performed using NEURO three-dimensional software (Siemens AG). Acquisition parameters were optimized to provide the best signal-to-noise ratio for the estimation of diffusion tensors. All data were trans- ferred to an independent workstation and were analyzed using FUNCTOOL software (GE Healthcare Life Sciences, Chalfont, UK) provided by the manufacturer. Qualitative assessment of all images was performed to evaluate the MS findings. DEC, FA, diffusion-weighted imaging (DWI) and ADC were measured in multiple MS plaques, regardless of their size, and in the symmetrical NAWM. For the analysis, a round-shape of $2.034 \mathrm{~mm}$ diameter was placed in the region of interest (ROI). Four specific regions were measured: i) the center of the MS lesions located in the silent lesion location; ii) the subacute phase of the lesion; iii) NAWM (the corpus callosum was selected); and iv) the deep brain gray matter (DBGM) (the thalamus and caudate nucleus were selected). In the normal controls, the corpus callosum, thalamus and caudate nucleus were analyzed. The FA, MD, $\lambda / /$ and $\lambda \perp$ values were measured and recorded.

Following the acquisition of DTI data, the data was processed to analyze the parameters of the diffusion using diffusion tensor tractography (DTT). For this analysis, a diffusion tensor matrix was generated for each voxel acquired using DWI. The RGB (red-green-blue) color-coded scheme attributes a color for each orientation of the fibers. Fibers crossing from left to right are visualized in red, fibers crossing anteriorly to posteriorly are visualized in green, and fibers crossing inferiorly to superiorly are visualized in blue. All fibers of the MS brain were studied based on the indicated ROI and computer program analysis.

Statistical analysis. Statistical analysis was performed by employing SPSS 17.0 statistical analysis software (SPSS, Inc., Chicago, IL, USA). Results are expressed as the mean \pm standard deviation. Individual comparisons of two sample groups were performed by independent t-test. Comparison of multiple sample groups were performed by single-factor analysis of variance. Prior to the test, Levene's test was used to test the homoscedasticity of the sample. Where necessary, Welch, least significant difference and Tamhane's tests were also applied. $\mathrm{P}<0.05$ was considered to indicate a statistically significant difference.

\section{Results}

Subjects. Ten patients with MS (female, 8; male; 2) were enrolled in the present study between September 2011 to June 2012. McDonald criteria was used for diagnosing the patients with MS, and all patients had relapsing-remitting MS. Eight of these patients had lesions in both their brains and spinal cords, whereas the remaining two only exhibited lesions in their brains. Patient ages ranged from 27 to 55 years, with a mean age of 37.3 years. Ten sex- and age-matched healthy volunteers were enrolled as normal controls.

Comparison of MS lesions, NAWM, and normal white matter. Significant differences in FA, MD, $\lambda / /$ and $\lambda \perp$ values were detected among silent lesions, subacute lesions, NAWM and white matter of normal controls $(\mathrm{P}<0.05)$. In particular, the FA and $\lambda / /$ values of silent and subacute lesions were lower than those of NAWM and normal controls ( $\mathrm{P}>0.05)$; in contrast, the MD and $\lambda \perp$ values of silent and subacute lesions were higher than those of NAWM and control group (P>0.05). FA values of NAWM were lower than that of the normal control 
Table I. FA, MD, $\lambda / /$ and $\lambda \perp$ values in all regions of interest (unitx $10^{-3} \mathrm{~mm}^{2} / \mathrm{s}$ ).

\begin{tabular}{lcccc}
\hline & FA & MD & $\lambda / /$ & $\lambda \perp$ \\
1 & $0.245 \pm 0.015$ & $1.214 \pm 0.341$ & $1.517 \pm 0.334$ & $1.058 \pm 0.377$ \\
2 & $0.227 \pm 0.093$ & $0.922 \pm 0.279$ & $1.131 \pm 0.272$ & $0.817 \pm 0.284$ \\
3 & $0.779 \pm 0.098$ & $0.789 \pm 0.085$ & $1.718 \pm 0.168$ & $0.324 \pm 0.121$ \\
4 & $0.863 \pm 0.059$ & $0.705 \pm 0.048$ & $1.699 \pm 0.153$ & $0.208 \pm 0.069$ \\
P-value & $<0.001$ & $<0.001$ & $<0.001$ & $<0.001$ \\
P1-2 & 0.999 & 0.123 & 0.029 & $<0.322$ \\
P1-3 & $<0.001$ & $<0.001$ & 0.053 & $<0.001$ \\
P1-4 & $<0.001$ & $<0.001$ & 0.094 & 0.005 \\
P2-3 & $<0.001$ & 0.726 & 0.002 & 0.001 \\
P2-4 & $<0.001$ & 0.259 & 0.002 & $<0.001$ \\
P3-4 & $<0.001$ & $<0.001$ & 0.988 & \\
\hline
\end{tabular}

FA, fractional anisotropy; MD, mean diffusivity; 1, silent lesions; 2, subacute lesions; 3, normal appearing white matter; 4, normal white matter.
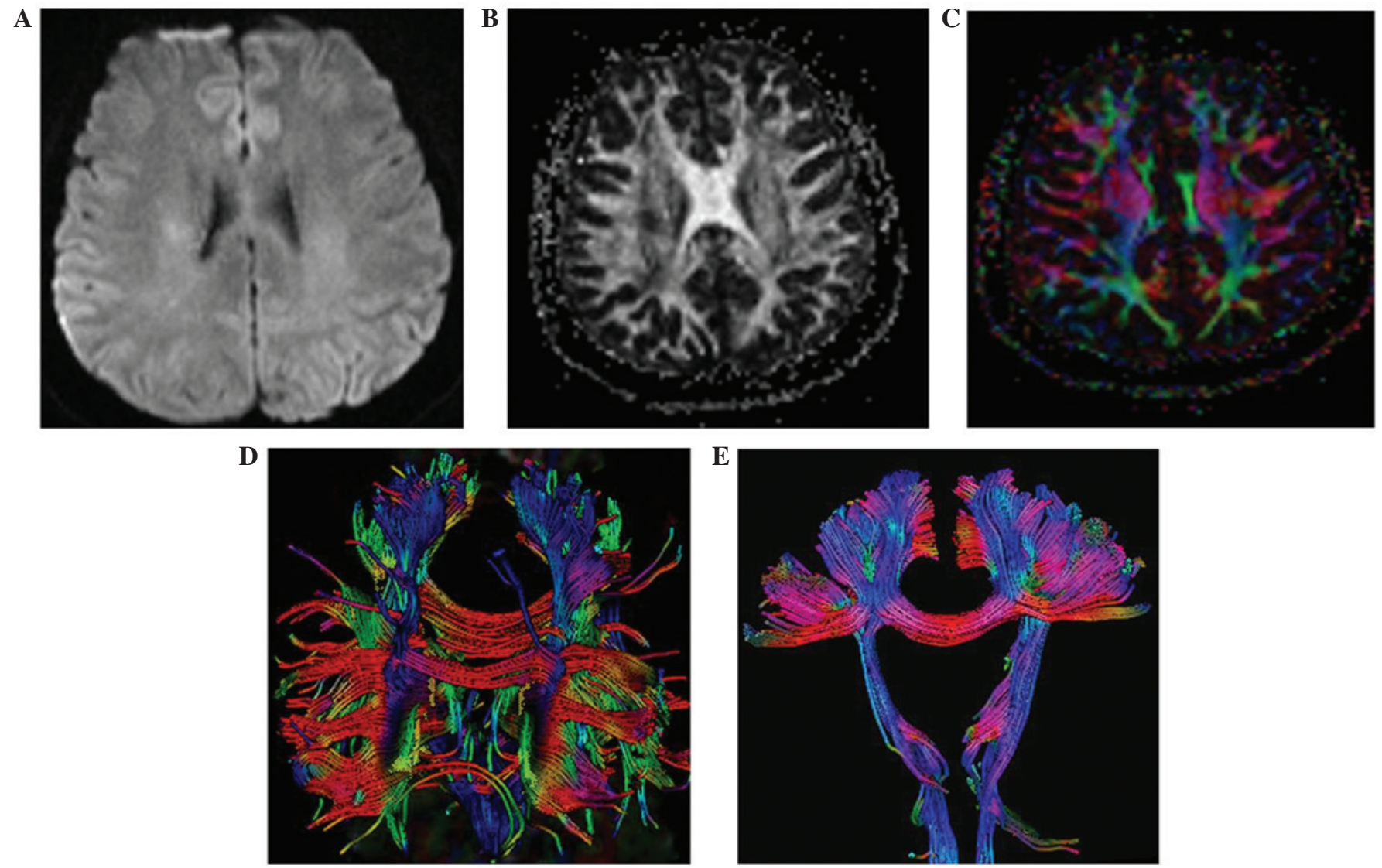

Figure 1. Images from a 27 year old patient admitted with slurred speech that lasted for one week. (A) Diffusion-weighted imaging showing right corona radiate abnormal signal. (B) FA illustrating the reduced signal at the right side of the lesion as compared with the surrounding normal tissue. (C) Directionally-encoded color map indicating that the signal at the right lesion was significantly reduced. (D) As shown using FA, bilateral corona radiata tracking ROIs fiber bundles in the corticospinal tract fibers corresponding to the fiber interruption and multiple sclerosis lesions. (E) Indicating the ROIs fibers of the lesions, the interruption of reconstructed fibers is evident at site of the lesion, with fewer fibers at the lesion site as compared with the contralateral side. FA, fractional anisotropy.

( $\mathrm{P}>0.05)$; however, $\mathrm{MD}$ and $\lambda \perp$ values were higher than those of the control group $(\mathrm{P}>0.05)$. Further findings identified that there were no significant differences in FA, MD and $\lambda \perp$ values between silent and subacute lesions ( $\mathrm{P}>0.05)$. Furthermore, there was no significant difference in $\lambda / /$ values among silent lesions, NAWM and the normal controls $(\mathrm{P}>0.05)$, and there were no significant differences in $\mathrm{MD}$ values between subacute lesions, NAWM and normal controls (P>0.05; Table I). An example from a 27-year-old patient who was admitted with slurred speech that lasted for one week is shown in Fig. 1. DWI showed that the right corona radiated an abnormal signal (Fig. 1A). Using FA, the MS lesions appeared as a region of relatively reduced hyperdensity, as compared with the hyperdensity signal of surrounding NAWM (Fig. 1B). Notably, the lesion was 
Table II. FA, MD, $\lambda / /$ and $\lambda \perp$ values in different regions of interest in MS and normal brains (unitx $10^{-3} \mathrm{~mm}^{2} / \mathrm{s}$ ).

\begin{tabular}{lcccc}
\hline & FA & MD & $\lambda / /$ & $\lambda \perp$ \\
\hline 1 & $0.346 \pm 0.091$ & $0.743 \pm 0.076$ & $1.068 \pm 0.241$ & $0.601 \pm 0.094$ \\
2 & $0.164 \pm 0.043$ & $0.729 \pm 0.145$ & $0.873 \pm 0.080$ & $0.693 \pm 0.081$ \\
3 & $0.329 \pm 0.037$ & $0.696 \pm 0.069$ & $0.932 \pm 0.079$ & $0.576 \pm 0.068$ \\
4 & $0.194 \pm 0.045$ & $0.702 \pm 0.067$ & $0.840 \pm 0.081$ & $0.632 \pm 0.069$ \\
5 & $0.255 \pm 0.116$ & $0.737 \pm 0.115$ & $0.971 \pm 0.204$ & $0.647 \pm 0.098$ \\
6 & $0.262 \pm 0.080$ & $0.699 \pm 0.067$ & $0.886 \pm 0.092$ & $0.604 \pm 0.074$ \\
t1-5 & 0.751 & 2.066 & 2.397 & 0.959 \\
P1-3 & 0.459 & 0.046 & 0.022 & 0.343 \\
t2-4 & -2.117 & 0.769 & 1.298 & 2.596 \\
P2-4 & 0.041 & 0.446 & 0.202 & 0.013 \\
t5-6 & -0.292 & 1.786 & 2.400 & 2.226 \\
P5-6 & 0.771 & 0.078 & 0.019 & 0.029 \\
\hline
\end{tabular}

MS, multiple sclerosis; FA, fractional anisotropy; MD, mean diffusivity; 1 , MS thalamus; 2 , MS caudate nucleus; 3 , normal thalamus; 4, normal caudate nucleus; 5 , MS deep brain gray matter; 6 , normal deep brain grey matter.

not noticeable when the DWI method was used, whereas the lesion can be visualized clearly using FA (Fig. 1A and B). DEC revealed a reduced color signal in the lesion fiber (Fig. 1C), and DTT fiber tractography indicated that the fiber was broken in MS plaques, which is known as the 'plaque avoid' phenomenon (8) (Fig. 1D), indicating that there were fewer fibers downstream of the lesion compared with the contralateral side (Fig. 1E).

DBGM of patients with MS and deep gray matter of normal controls. FA, MD, $\lambda / /$ and $\lambda \perp$ values in the thalamus were significantly increased in patients with MS as compared with those of normal patients $(\mathrm{P}<0.05)$. In the caudate nucleus and deep brain gray matter, the FA values were lower in patients with MS as compared with those of normal controls $(\mathrm{P}>0.05)$; whereas MD, $\lambda / /$ and $\lambda \perp$ values were higher. Furthermore, the FA and $\lambda \perp$ values were significantly different in the caudate nucleus between patients with MS and normal controls $(\mathrm{P}<0.05)$. There was a significant increase in $\lambda / /$ and $\lambda \perp$ values in the MS deep gray matter, as compared with normal gray matter $(\mathrm{P}<0.05$; Table II).

\section{Discussion}

MS, which is a demyelinating disease of the central nervous system, is the most common cause of non-traumatic neurological disability in young adults. Conventional MRI has limitations in the identification of MS lesions; however, novel neuroradiologic techniques and measurements are emerging that may offer a improved estimation of disease status and the amplitude of injuries. One of these new methods, magnetic resonance DTI, has been demonstrated to improve understanding of the cerebral white matter configuration and the different pathologies that may influence it (5). In the present study, measurements from DTI, including MD, FA, $\lambda / /$, and $\lambda \perp$ values, were used to study MS lesions, NAWM and the white matter of normal controls. Significant differences were detected in the FA, MD, $\lambda / /$ values among silent lesions, subacute lesions, NAWM and normal white matter.
Specifically, the FA and $\lambda / /$ values in silent lesion and subacute lesions were lower, whereas MD and $\lambda \perp$ values in silent lesions and subacute lesions were higher than those of NAWM and normal controls. Theoretically, FA represents the degree of directionality of diffusion in the studied tissue, and MD indicates the degree of diffusion of water molecules. The $\lambda / /$ and $\lambda \perp$ values indicate the diffusion parallel to the nerve fibers and vertical direction to the nerve fibers, respectively. The reduction of FA values correlates with axonal injury and the destruction of myelin integrity. Increasing MD values are associated with infection and tissue edema (9). MS pathological changes reduce the resistance of water molecules, thereby increasing the degree of dispersion, which correlates to an increase in the MD, $\lambda / /$ and $\lambda \perp$ values and a decrease in the FA values within MS plaques (5). These findings are consistent with pathological study results from MS autopsy and biopsy cases as published by Barkhof et al (1), Lucchineeti et al (10), Storch et al (11).

Previous studies have suggested that $\lambda / /$ values are associated with axonal degeneration $(12,13)$, whereas $\lambda \perp$ values are related to demyelination, edema, and proliferation of neuroglial cells $(12,13)$. Using a mouse model of MS, it was demonstrated that an increased $\lambda \perp$ value reflected demyelination of the corpus callosum (14). Furthermore, in an animal model of retinal ischemia, $\lambda / /$ value reduction correlated with axon damage (14). These data suggests that the decrease in the FA value of NAWM and the increase in MD, $\lambda / /$ and $\lambda \perp$ values as compared to normal controls in the present study indicates the presence of occult damage to the NAWM area. The present findings are also consistent with in vivo data from Trip et al (15), who found that mean MD was elevated and mean FA was reduced in patients with optic neuritis, as compared with normal controls. This suggests that the pathological change in optic neuritis is similar to MS, as indicated by the present findings. Notably, these findings suggest that DTI measurements provide an indication of the structural integrity of axons.

The present study found that $\lambda / /$ values in silent lesions are similar to that of NAWM and normal controls, whereas 
the MD values of subacute lesions are close to that of NAWM and normal controls. This phenomenon may be associated with remyelination. MS lesions are composed of remyelination, astrocytes and oligodendrocytes regeneration (16-20), which act as a barrier for water molecule movement, resulting in a decrease in the degree of dispersion and the reduction of the MD and $\lambda / /$ values. Pulizzi et al (21) found that T1WI hypodensity of MS lesions can be changed into isodensity during the evolvement of lesions, which may also be related to remyelination. It has been proposed that damaged tissue and surrounding astrocytes are able to release molecules that mediate a rapid microglial response towards injury $(16-18,22)$. The increased FA values observed in the present study are relevant to the response of astrocytes to local injury of MS lesions, and supports the hypothesis that remyelination occurs in MS lesions.

The corpus callosum is the largest white matter tract in the brain, connecting the two brain hemispheres. It has an important role in movement, and the sensory and cognitive function between two hemispheres. It is also important in the behavioral and cognitive functions of patients with MS. The corpus callosum was selected as the area of normal white matter as it has a strong anisotropy with a high FA value. The present findings indicated that when white matter is involved in MS lesions, there is a reduction of hyperdensity of MS lesions on MRI, which has sharp contrast with surrounding white matter.

DEC uses a RGB color-coded scheme and attributes a color for each orientation of the fibers, such that fibers crossing from left to right are visualized in red, fibers crossing anteriorly to posteriorly are visualized in green, and fibers crossing inferiorly to superiorly are visualized in blue. In addition, FA allows for the visualization of lesions more clearly and larger than with DWI. The application of DTT may aid in the identification of broken fibers and the diminishment of distal fibers in MS lesions, which are associated with vasogenic edema, demyelination, axonal degenetation and other pathological changes related to disintegrity of the nerve fibers $(13,14)$. No obvious abnormality of NAWM was detected by FA, DEC or DTT in the present study. This may be due to the pathological alterations in NAWM not being significant enough to be detected. Therefore, for optimal pathological diagnosis and study of patients with MS, we propose that FA, MD, $\lambda / /$ and $\lambda \perp$ values should be utilized in combination.

To date, few studies have focused on the role of DTI in deep brain gray matter in patients with MS, and the results remain controversial (23-25). The present DTI study found that $\mathrm{FA}, \mathrm{MD}, \lambda / /, \lambda \perp$ values in the thalamus were increased in patients with MS, compared with those of normal patients. Furthermore, the $\lambda / /, \lambda \perp$ and MD values were increased and the FA values were decreased in the caudate nucleus and deep brain gray matter of patients with MS, as compared with those of normal controls. The $\lambda / /$ and $\lambda \perp$ values are also increased in the lesions of patients with MS, as compared with those of deep gray matter. The present finding of increased FA and $\mathrm{MD}$ values in the thalamus are consistent with previous studies, including a report by Tovar-Moll et al (26), which suggested that an increase in the FA values in the thalamus correlates with thalamus reactive reconstruction (26-28).
Future studies addressing the relationship between DTI and different subtypes of MS $(10,11,19,20)$, particularly with progressive MS, and a comparison to histopathology, are necessary to elucidate the exact pathological correlation between white matter to gray matter and its involvement in patients with MS.

\section{Acknowledgements}

The authors would like to thank Hongwei Zhou (The First Teaching Hospital of the Jilin University) for technical advice on the neuroradiological images.

\section{References}

1. Balcer LJ: Clinical practice. Optic neuritis. N Engl J Med 354: 1273-1280, 2006.

2. Shams PN and Plant GT: Optic neuritis: A review. Int MS J 16: 82-89, 2009.

3. Heesen C, Kasper J, Segal J, Köpke S and Mühlhauser I: Decisional role preferences, risk knowledge and information interests in patients with multiple sclerosis. Mult Scler 10: 643-650, 2004.

4. Barkhof F: The clinico-radiological paradox in multiple sclerosis revisited. Curr Opin Neurol 15: 239-245, 2002.

5. Beaulieu C: The basis of anisotropic water diffusion in the nervous system-a technical review. NMR Biomed 15: 435-455, 2002.

6. Yamout B, Alroughani R, Al-Jumah M, Khoury S, Abouzeid N, Dahdaleh M, Alsharoqi I, Inshasi J, Hashem S, Zakaria M, et al: Consensus guidelines for the diagnosis and treatment of multiple sclerosis. Curr Med Res Opin 29: 611-621, 2013.

7. Szeszko PR, Vogel J, Ashtari M, Malhotra AK, Bates J, Kane JM, Bilder RM, Frevert T and Lim K: Sex differences in frontal lobe white matter microstructure: A DTI study. Neuroreport 14: 2469-2473, 2003.

8. Kealey SM, Kim Y, Whiting WL, Madden DJ and Provenzale JM: Determination of multiple sclerosis plaque size with diffusion-tensor MR imaging: Comparison study with healthy volunteers. Radiology 236: 615-620, 2005.

9. Ciccarelli O, Werring DJ, Barker GJ, Griffin CM, Wheeler-Kingshott CA, Miller DH and Thompson AJ: A study of the mechanisms of normal appearing white matter damage in multiple sclerosis using diffusion tensor imaging-evidence of Wallerian degeneration. J Neuro1 250: 287-292, 2003.

10. Lucchinetti CF, Brück W and Noseworthy J: Multiple sclerosis: Recent developments in neuropathology, pathogenesis, magnetic resonance imaging studies and treatment. Curr Opin Neurol 14: 259-269, 2001.

11. Storch MK, Piddlesden S, Haltia M, Iivanainen M, Morgan P and Lassmann H: Multiple sclerosis: In situ evidence for antibody- and complement-mediated demyelination. Ann Neurol 43: 465-471, 1998.

12. Kolbe S, Bajraszewski C, Chapman C, Nguyen T, Mitchell P, Paine M, Butzkueven H, Johnston L, Kilpatrick T and Egan G: Diffusion tensor imaging of the optic radiations after optic neuritis. Hum Brain Mapp 33: 2047-2061, 2012.

13. Zhang J, Jones M, DeBoy CA, Reich DS, Farrell JA, Hoffman PN, Griffin JW, Sheikh KA, Miller MI, Mori S and Calabresi PA: Diffusion tensor magnetic resonance imaging of Wallerian degeneration in rat spinal cord after dorsal root axotomy. J Neurosci 29: 3160-3171, 2009.

14. Song SK, Sun SW, Ju WK, Lin SJ, Cross AH and Neufeld AH: Diffusion tensor imaging detects and differentiates axon and myelin degeneration in mouse optic nerve after retinal ischemia. Neuroimage 20: 1714-1722, 2003.

15. Trip SA, Wheeler-Kingshott C, Jones SJ, Li WY, Barker GJ, Thompson AJ, Plant GT and Miller DH: Optic nerve diffusion tensor imaging in optic neuritis. Neuroimage 30: 498-505, 2006.

16. Lucchinetti CF, Brück W, Parisi J, Scheithauer B, Rodriguez M and Lassmann H: Heterogeneity of multiple sclerosis lesions: Implications for the pathogenesis of demyelination. Ann Neurol 47: 707-717, 2000. 
17. Lucchinetti CF, Bruck W and Lassmann H: Evidence for pathogenic heterogeneity in multiple sclerosis. Ann Neurol 56: 308, 2004.

18. Barnett $\mathrm{MH}$ and Prineas JW: Relapsing and remitting multiple sclerosis: Pathology of the newly forming lesion. Ann Neurol 55: 458-468, 2004.

19. Weinshenker BG: The natural history of multiple sclerosis. Neurol Clin 13: 119-146, 1995.

20. Lucchinetti CF, Bruck W and Lassmann H (eds): Pathology and pathogenesis of multiple sclerosis, 2nd edititon. Elsevier Science, USA, 2003.

21. Pulizzi A, Rovaris M, Judica E, Sormani MP, Martinelli V, Comi G and Filippi M: Determinants of disability in multiple sclerosis at various disease stages: A muitiparametric magnetic resonance study. Arch Neurol 64: 1163-1168, 2007.

22. Davalos D, Grutzendler J, Yang G, Kim JV, Zuo Y, Jung S, Littman DR, Dustin ML and Gan WB: ATP mediates rapid microglial response to local brain injury in vivo. Nat Neurosci 8: 752-758, 2005.

23. Griffin CM, Chard DT, Ciccarelli O, Kapoor B, Barker GJ, Thompson AI and Miller DH: Diffusion tensor imaging in early relapsing-remitting multiple sclerosis. Mult Scler 7: 290-297, 2001
24. Filippi M, Bozzali M and Comi G: Magnetization transfer and diffusion tensor MR imaging of basal ganglia from patients with multiple sclerosis. J Neurol Sci 183: 69-72, 2001

25. Steenwijk MD, Daams M, Pouwels PJ, Balk LJ, Tewarie PK, Killestein J, Uitdehaag BM, Geurts JJ, Barkhof F and Vrenken $\mathrm{H}$ : What explains gray matter atrophy in long-standing multiple sclerosis? Radiology 272: 832-842, 2014.

26. Tovar-Moll F, Evangelou IE, Chiu AW, Richert ND, Ostuni JL, Ohayon JM, Auh S, Ehrmantraut M, Talagala SL, McFarland HF and Bagnato F: Thalamic involvement and its impact on clinical disability in patients with multiple sclerosis: A diffusion tensor imaging study at 3T. AJNR Am J Neuroradiol 30: 1380-1386, 2009.

27. Kipp M, Wagenknecht N, Beyer C, Samer S, Wuerfel J and Nikoubashman O: Thalamus pathology in multiple sclerosis: From biology to clinical application. Cell Mol Life Sci 72: 1127-1147, 2015.

28. Sorgun MH, Yucesan C and Tegin C: Is malnutrition a problem for multiple sclerosis patients? J Clin Neurosci 21: 1603-1605, 2014. 